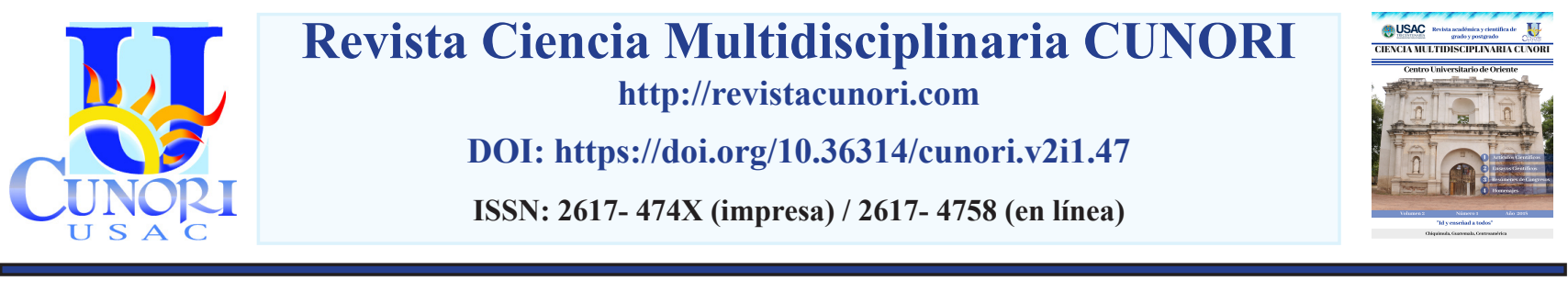

Como citar el artículo

Xuyá M., Chicas R. \& Mazariegos, E. (2018). Eficacia de atención prenatal en pacientes que consultan al Hospital Regional de Zacapa. Revista Ciencia Multidisciplinaria CUNORI, 2(1), 17-21. https://doi.org/10.36314/cunori.v2i1.47

\title{
Eficacia de atención prenatal en pacientes que consultan al Hospital Regional de Zacapa
}

\section{Efficacy of prenatal care in patients who consult Zacapa Regional Hospital}

\author{
Marco Xuyá*, Otto Chicas \& Edvin Mazariegos \\ Centro Universitario de Oriente -CUNORI, Universidad de San Carlos de Guatemala \\ Recibido: 05 de abril de 2018 Aceptado: 03 de agosto de 2018 \\ Disponible en internet el 17 de agosto de 2018
}

\author{
*Autor para correspondencia. \\ Correo electrónico: marco.xuya.med@gmail.com
}

Resumen

$\mathrm{L}$ a atención prenatal es una medida eficaz y de bajo costo, para disminuir los índices de mortalidad materno-infantil, para lograrlo, la atención debe ser periódica, extensa, y continua. Lográndose medir a través de índices, entre ellos se tiene, al índice de Kotelchuck. Lo que se da a conocer es un estudio descriptivo retrospectivo, sobre la eficacia de la atención prenatal en mujeres que consultaron la emergencia del Hospital Regional de Zacapa. La muestra fue de 632 pacientes con intervalo de confianza del 99\%, dicha muestra se obtuvo del universo de 19,214 pacientes de enero de 2012 a diciembre de 2016. Se utilizaron tres criterios de inclusión: mayor de edad, trabajo de parto activo y embarazo a término. Se observó que el 37\% (235) de los pacientes tuvieron una atención prenatal inadecuada, el 25\% (156) llevaron atención prenatal intermedia, al mismo tiempo 25\% (158) con atención prenatal adecuada y únicamente el 13\% (83) obtuvieron una atención adecuada plus. Determinándose que el 62\% de las pacientes no alcanzaron con las visitas mínimas requeridas para tener una atención prenatal adecuada o no logran iniciar su atención prenatal en los primero cuatro meses de gestación. Lo cual puede repercutir en la tasa de mortalidad materno-infantil en Guatemala.

Palabras clave: atención prenatal, eficacia de atención prenatal, índice de Kotelchuck, mortalidad materno-infantil

Abstract

Drenatal care is an effective and inexpensive measure to reduce maternal and infant mortality rates, but in order to achieve this, attention must be periodic, extensive, and continuous. Being able to measure itself through several indices, among them we have, at the Kotelchuck index. This is a retrospective, descriptive study on the effectiveness of prenatal care in women who consulted the emergency of the Regional Hospital of Zacapa. The sample is of 632 patients with a confidence interval of $99 \%$, this sample was obtained from the universe of 19,214 patients from January 2012 to December 2016. Three inclusion criteria were used: adult age, active labor and pregnancy at finished. It was observed that 37\% (235) of the patients had inadequate prenatal care, $25 \%$ (156) took intermediate prenatal care, at the same time $25 \%$ (158) with adequate prenatal care and only $13 \%$ (83) obtained adequate attention plus. Determining that $62 \%$ of the patients do not reach the minimum visits required to have adequate prenatal care or fail to start their prenatal care in the first four months of gestation. This may have an impact on the maternal and infant mortality rate in Guatemala.

Keywords: prenatal care, effectiveness of prenatal care, Kotelchuck index, maternal and infant mortality 


\section{Introducción}

La Organización Mundial de la Salud define atención prenatal como el cuidado proporcionado por profesionales de la salud a las mujeres para garantizar las mejores condiciones de salud tanto para la madre y el feto durante el embarazo. (WHO, 2016). Este programa incluye: atención previa a la concepción, diagnóstico oportuno del embarazo, valoración prenatal inicial y consultas prenatales de seguimiento (Cunningham et al., 2010). Siendo los elementos estándar de la atención prenatal: examen físico de rutina (incluido un examen de pelvis) en la visita inicial, peso materno y presión arterial en todas las visitas, auscultación fetal de frecuencia cardíaca después de la 10 a 12 semanas con un monitor Doppler o después de 20 semanas con un estetoscopio, altura del fondo uterino después de 20 semanas y posición fetal a partir de las 36 semanas. (Zolotor y Carlough, 2014). También se ve la necesidad de algunas pruebas de laboratorio como: papanicolaou, grupo sanguíneo y factor Rh, glicemia, hemograma, análisis de orina, serología para sífilis, Chagas, toxoplasmosis, VIH y citomegalovirus. (Schwarcz et al., 1986)

Denominándose atención prenatal eficiente a la capacidad de disponer de alguien o de algo para conseguir un efecto determinado es el que cumple con cuatro requisitos básicos; precoz o temprana, periódico o continúo, completo o integral y extenso o de amplia cobertura. (Schwarcz et al., s.f.). Son barreras para el control prenatal efectivo: a) su costo, cuando no es gratuito para la usuaria, a lo que se debe agregar gastos de transporte, pérdida de horas laborales, etc., b) inadecuada capacidad (calidad, tiempo, etc.) del equipo de salud, c) problemas en la organización para brindar el control prenatal, d) barreras de orden cultural, e) inaccesibilidad geográfica, f) descreimiento en las bondades del sistema de salud y en la necesidad de control, g) falta de promoción en las comunidades y apropiación de su importancia (Fescina et al., 2010).

Utilizándose índices para la medición de la eficacia entre ellos se encuentra el índice de Kotelchuck el cual mide la eficacia mediante la medición de dos componentes distintos de atención prenatal: inicio de la atención prenatal (adecuación de la iniciación) y número de visitas recibidas durante la gestación (adecuación de los servicios recibidos) (Kotelchuck, 1994). En Guatemala durante el año 2016 se obtuvo una cobertura de 96.2\% según datos de la Encuesta Nacional de Salud Materno Infantil, la cual puede considerarse alta, siendo importante aclarar que las encuestas reportan la cobertura, como al menos una cita en alguno de los tres trimestres, desconociendo por completo las cifras de eficacia de la atención prenatal debido a que se carece de estudios a nivel nacional, siendo estos datos necesarios para una evaluación objetiva del programa de salud materna en Guatemala.

\section{Material y métodos}

Se tomó para el estudio una muestra representativa de 632 pacientes con un intervalo de confianza del $99 \%$, el objeto de la investigación lo constituyeron los expedientes de pacientes que consultaron con trabajo de parto activo a la emergencia del Hospital Regional de Zacapa de enero de 2012 a diciembre de 2016, se utilizaron tres criterios de inclusión: trabajo de parto activo, mayor de edad y embarazo a término. 
Para la recolección de datos se revisaron las fichas clínicas de todos los pacientes que cumplieron los criterios de inclusión durante el período establecido, anotándose la edad gestacional al que iniciaron la atención prenatal y el número de visitas que tuvieron durante la gestación; esta información se registró en la boleta de recolección de datos. Los datos recolectados se ingresaron a una base de datos en Excel, según el orden establecido en las técnicas e instrumentos de recolección; luego fueron tabulados y expresados gráficamente.

\section{Resultados}

Eficacia de la atención prenatal en relación al inicio de la atención prenatal. El 36\% (226) de las pacientes tuvieron una atención prenatal adecuada con relación a la adecuación del inicio de la atención prenatal, el 29\% (184) presentaron una atención prenatal adecuada plus, 20\% (127) de las pacientes tuvo una atención prenatal inadecuada, por último el 15\% (95) tuvo una atención prenatal intermedia.

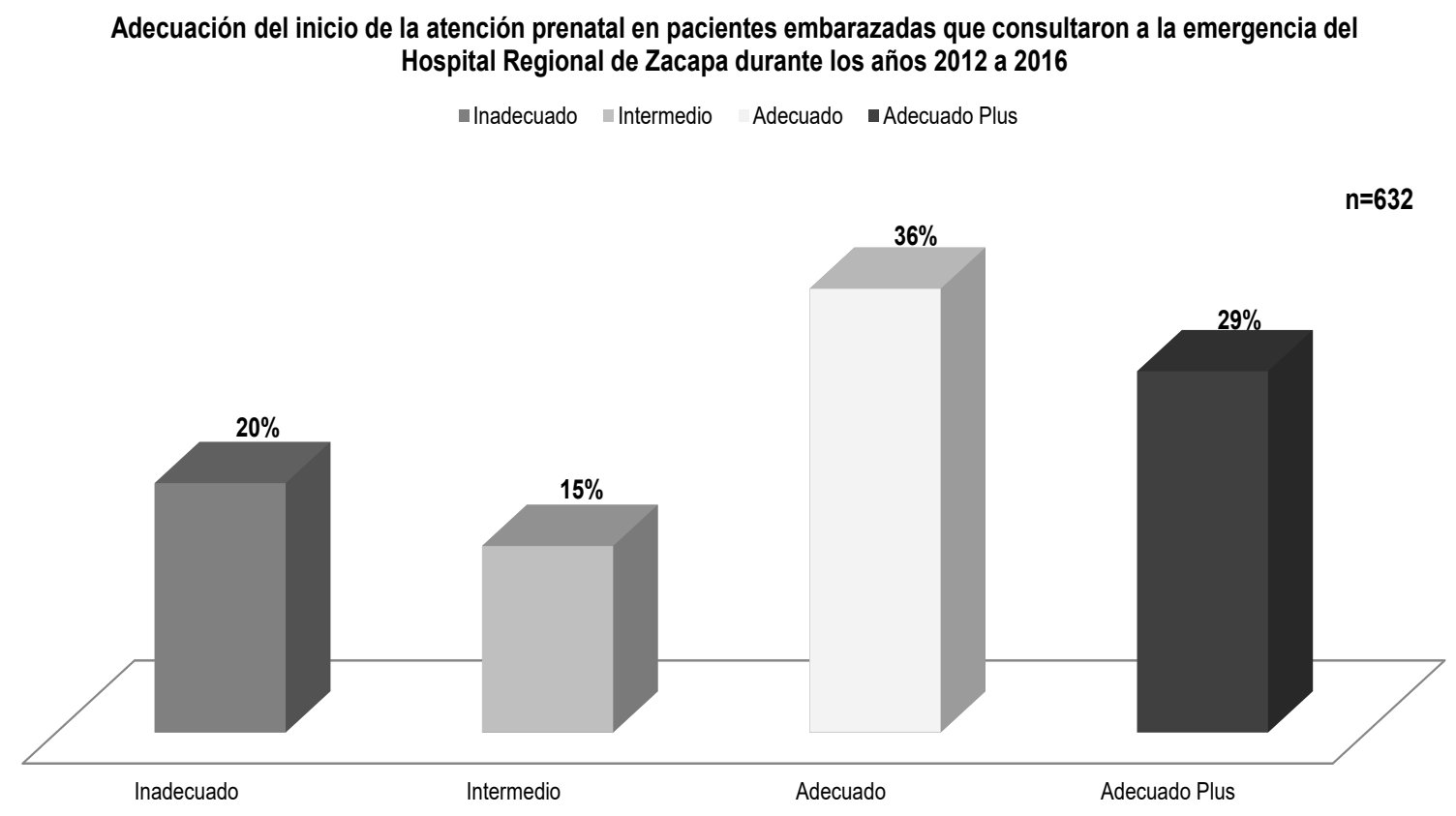

Figura 1. Adecuación del inicio de la atención prenatal en pacientes embarazadas que cosultaron a la emergencia del Hospital Regional de Zacapa durante los años 2012 a 2016.

Eficacia de la atención prenatal en relación al número de visitas recibidas durante la atención prenatal. Con respecto a la adecuación de las visitas recibidas el 32\% (200) de las pacientes tuvieron una atención prenatal inadecuada, 29\% (185) obtuvo una atención prenatal intermedia, 26\% (163) tuvo una atención prenatal adecuada y únicamente el 13\% (84) llevó una atención prenatal adecuada plus. 
Adecuación de las visitas recibidas en pacientes embarazadas que consultaron a la emergencia del Hospital Regional de Zacapa durante los años 2012 a 2016.

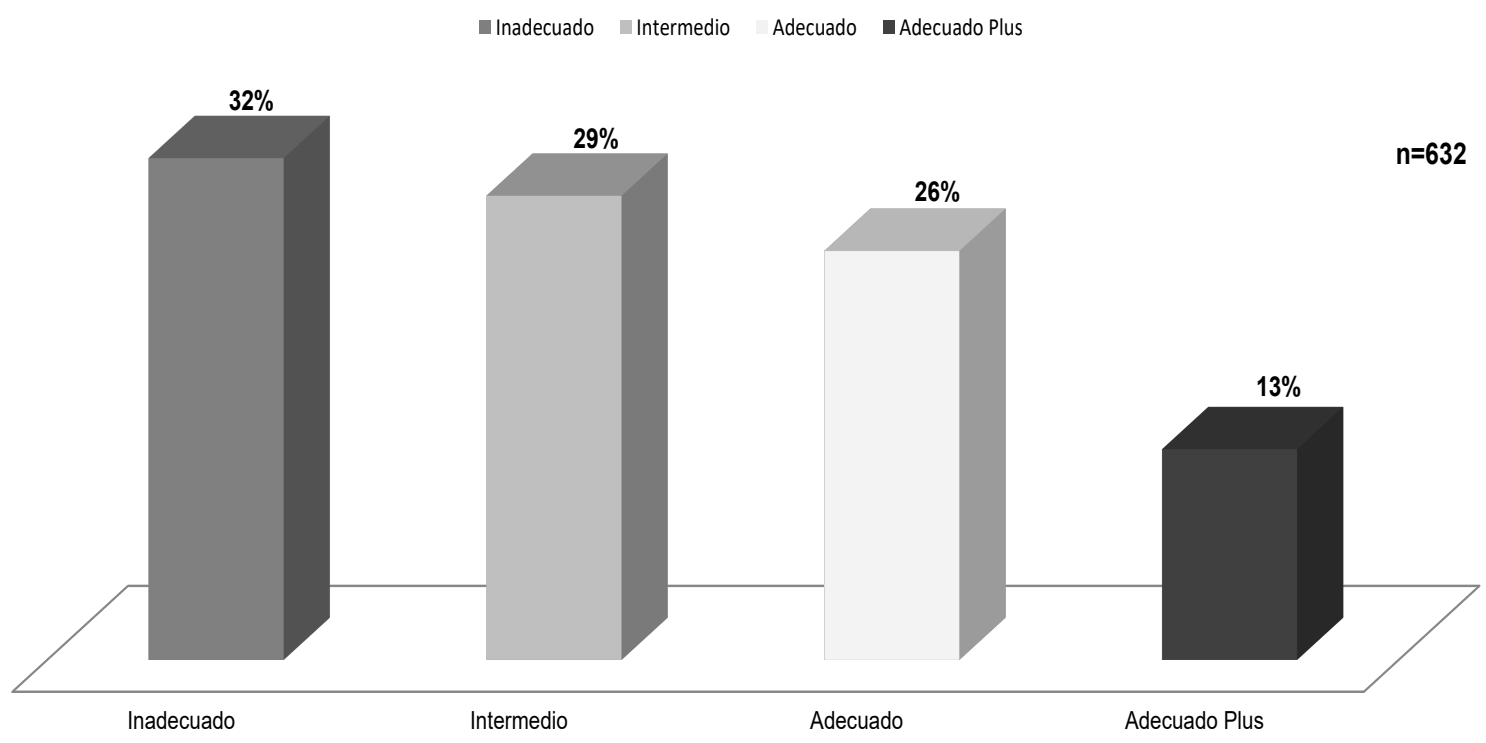

Figura 2. Adecuación de las visitas recibidas en pacientes embarazadas que consultaron a la emergencia del Hospital Regional de Zacapa durante los años 2012 a 2016.

En la eficacia global de la atención prenatal el 37\% (235) de las pacientes llevó una atención prenatal inadecuada, 25\% (158), tuvo una atención prenatal adecuada, 25\% (156) una atención prenatal intermedia, por último solamente el 13\% (83) obtuvo una atención prenatal adecuada plus.

Eficacia global de la adecuación de la atención prenatal en pacientes embarazadas que consultaron a la emergencia del Hospital Regional de Zacapa durante los años 2012 a 2016.

- Inadecuado $\square$ Intermedio Adecuado $\quad$ Adecuado Plus

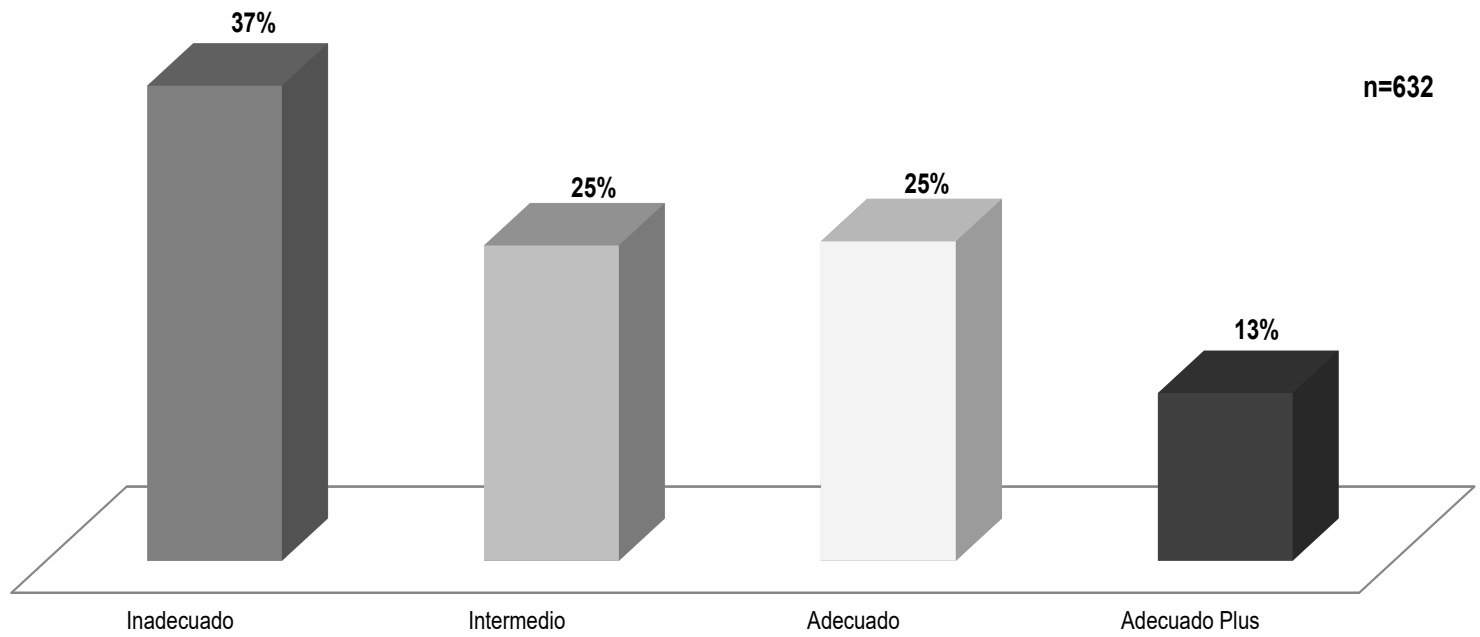

Figura 3. Eficacia global de la adecuación de la atención prenatal en pacientes embaraza- das que consultaron a la emergencia del Hospital Regional de Zacapa durante los años 2012 a 2016. 


\section{Discusión}

El 13\% (83 pacientes) de las pacientes a nivel global recibieron una atención prenatal adecuada plus, lo cual se ve reflejado en el 13\% (84 pacientes) que recibieron una atención prenatal adecuada plus en relación a la adecuación de las visitas recibidas. La mayoría de las pacientes obtuvo una atención prenatal adecuada 36\% (226 pacientes) y adecuada plus $29 \%$ (184 pacientes), con respecto a la adecuación del inicio de la atención prenatal, a pesar que la mayoría de pacientes tuvieron su primer contacto en el primer trimestre; no lograron continuar con las visitas requeridas para tener una atención prenatal adecuada o adecuada plus a nivel global. Demostrando que el $62 \%$ de los pacientes no cumplió con las visitas mínimas requeridas por la OMS, o la edad gestacional a la que iniciaron la atención prenatal no fue en los primeros 4 meses de gestación, por lo que no cumplen con los criterios de una atención prenatal eficiente, a pesar que la cobertura a nivel nacional es de aproximadamente $96.2 \%$.

\section{Agradecimientos}

Al personal del departamento de estadística del Hospital Regional de Zacapa por proporcionar la información necesaria para la realización de esta investigación.

\section{Referencias bibliográficas}

Cunningham, F., Leveno, K., Bloom, S., Hauth, J., Rouse, D. \& Spong, C., (2010), Williams Obstetricia, México DF, México: McGraw-Hill.

Fescina, R. H., de Mucio, B., Díaz Rossello, J. L., Martínez, G., Serruya, S., Durán, P., (2010), Salud sexual y reproductiva: guías para el continuo de atención de la mujer y el recién nacido focalizadas en APS, Montevideo, Uruguay: CLAP/SMR-OPS/OMS.

Kotelchuck, M., (1994). An evaluation of the Kessner adequacy of prenatal care index and a proposed adequacy of prenatal care utilization index. American Journal of Public Health. 84(9),1414-1420. https://doi.org/10.2105/AJPH.84.9.1414

Schwarcz, R., Díaz A. G., Fescina, R. H., de Mucio, B., Belitzky, R. \& Delgado L, R., (s.f.), Salud reproductiva materna perinatal: atención prenatal y del parto de bajo riesgo, Montevideo, Uruguay: CLAP-OPS/OMS.

Schwarcz, R. L., Duverges, C. A., Díaz, A. G. \& Fescina, R. H., (1986). Obstetricia. 4 ed, Buenos Aires, Argentina: El Ateneo.

World Health Organization. (2016). WHO recommendations on antenatal care for a positive pregnancy experience, Suiza.

Zolotor, A. J., Carlough \& M. C., (2014), Update on prenatal care, American Family Physician. 89(3), 199-208. 
Sobre el autor

\section{Marco Aarón Xuyá Castillo}

Médico y Cirujano egresado del Centro Universitario de Oriente CUNORI de la Universidad de San Carlos de Guatemala. Ha realizado investigaciones sobre "Nivel de calidad de atención en los servicios de medicina interna del Hospital Regional de Zacapa", "Estado al nacer de neonatos de pacientes con embarazo a término que recibieron esquema de maduración pulmonar", "Incidencia de apnea obstructiva del sueño en pacientes que consultan a la clínicas familiares del CUNORI", "Prevalencia del efecto Somogy en pacientes diagnosticados con Diabetes Mellitus 2", "Incidencia de hernias inguinales, femorales y recidivantes en los servicios de Cirugías en el Hospital Nacional Carlos Manuel Arana Osorio". Actualmente estudia el postgrado de Especialización en investigación en el Centro Universitario de Oriente, CUNORI.

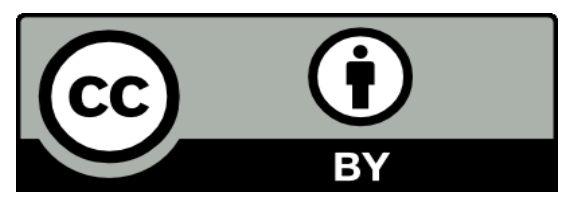

Este texto está protegido por una licencia CreativeCommons 4.0.

Usted es libre para compartir, copiar y redistribuir el material en cualquier medio o formato y adaptar el documento, remezclar, transformar y crear a partir del material para cualquier propósito, incluso comercialmente, siempre que cumpla la condición de atribución: usted debe reconocer el crédito de una obra de manera adecuada, proporcionar un enlace a la licencia, e indicar si se han realizado cambios. Puede hacerlo en cualquier forma razonable, pero no de forma tal que sugiera que tiene el apoyo del licenciante o lo recibe por el uso que hace. 